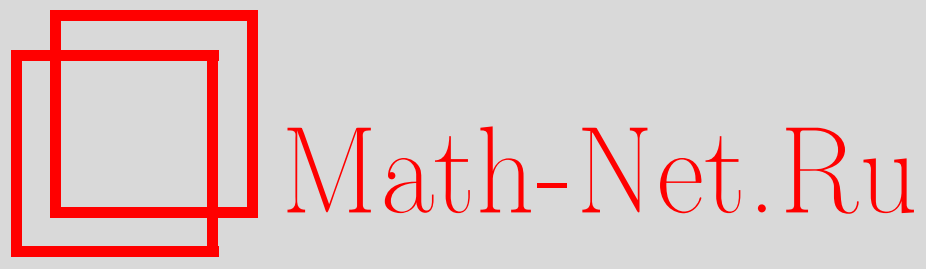

В. В. Доценко, Гомологии алгебры Ли векторных полей на прямой с коэффициентами в симметрических степенях ее присоединенного представления, Функи. анализ и его прил., 2006, том 40, выпуск 2, 13-19

DOI: https://doi.org/10.4213/faa3

Использование Общероссийского математического портала Math-Net.Ru подразумевает, что вы прочитали и согласны с пользовательским соглашением

http://www.mathnet.ru/rus/agreement

Параметры загрузки:

IP: 54.81 .137 .203

26 апреля 2023 г., 14:11:35

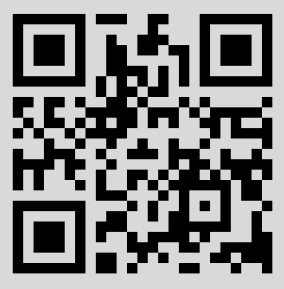


УДК 512.664.3

\title{
Гомологии алгебры Ли векторных полей на прямой с коэффициентами в симметрических степенях ее присоединенного представления*
}

\author{
(C) 2006. В. В. ДОцЕнко
}

\section{$\S 1$. Введение}

Пусть $W_{1}$ - алгебра Ли полиномиальных векторных полей на прямой, т. е. векторных полей вида $f(x) \frac{d}{d x}$, где $f(x) \in \mathbb{C}[x]$; стандартный базис в этой алгебре образуют векторные поля $e_{i}=x^{i+1} \frac{d}{d x}$ с коммутационными соотношениями $\left[e_{i}, e_{j}\right]=(j-i) e_{i+j}$. В настоящей статье вычислены гомологии алгебры $W_{1}$ с коэффициентами в модулях $S^{k} W_{1}$, т. е. симметрических степенях ее присоединенного представления. А именно, мы доказываем следующие результаты.

ОСНОВНАЯ ТЕОРЕМА. Пустъ $k>0$. Тогдa

(i) $H_{2}\left(W_{1}, \mathfrak{g l}_{1}, S^{k} W_{1}\right)=\mathbb{C}, H_{i}\left(W_{1}, \mathfrak{g l}_{1}, S^{k} W_{1}\right)=0$ nрu $i \neq 2$;

(ii) $H_{2}\left(W_{1}, S^{k} W_{1}\right)=H_{3}\left(W_{1}, S^{k} W_{1}\right)=\mathbb{C}, H_{i}\left(W_{1}, S^{k} W_{1}\right)=0$ nрu $i \neq 2,3$.

Это доказывает для $n=1$ гипотезу Фейгина и Цыгана [1] о гомологиях алгебры $W_{n}$ с коэффициентами в симметрических степенях ее присоединенного представления.

Вычисление этих гомологий основано на частичном вычислении гомологий (с теми же коэффициентами) подалгебры алгебры $W_{1}$, состоящей из векторных полей, имеющих нуль второго порядка в начале координат. (Эту алгебру обычно обозначают через $L_{1}$; базис в ней составляют элементы $e_{i}$ с $i \geqslant 1$. Более общо, через $L_{k}$ обозначается подалгебра, порожденная элементами $e_{i}$ с $i \geqslant k$.) В общем случае гомологии алгебры $L_{1}$ с нетривиальными коэффициентами вычислять сложнее, но в нашем случае можно доказать, что $L_{1}$-модули $S^{k} W_{1}$ в малых градуировках свободны. (Более точно это можно выразить так: существует (однородный) сюръективный гомоморфизм из свободного модуля над алгеброй $L_{1}$ на модуль $S^{k} W_{1}$, который на всех однородных компонентах достаточно малой степени имеет нулевое ядро; см. следствие 2 ниже.) Гомологии свободных модулей известны; если же модуль свободен в малых градуировках, то легко вычислить соответствующие однородные компоненты его гомологий. Способы же извлекать из сведений о гомологиях алгебры Ли $L_{1}$ информацию о гомологиях алгебры Ли $W_{1}$ с теми же коэффициентами вполне традиционны (см., например, [2]).

Структура работы такова. В первой части изучается строение симметрических степеней (как $L_{1}$-модулей) в малых градуировках. Это можно сделать средствами линейной алгебры (фактически речь идет о том, что матрицы некоторых операторов являются верхнетреугольными). Во второй части доказанные результаты перерабатываются в информацию о гомологиях алгебры $W_{1}$. Это

*Работа частично поддержана грантом Президента РФ №2044.2003.2 и грантом INTAS 03-3350. 
делается с помощью достаточно стандартных приемов гомологической алгебры. Завершает работу обсуждение случая тензорных степеней присоединенного представления; в частности, предлагается (предположительно почти точная) оценка снизу на соответствующие гомологии.

Я благодарен моему учителю Б. Л. Фейгину за постановку задачи и стимулирующие обсуждения. Также я благодарен рецензенту за многочисленные предложения по улучшению текста.

\section{§2. Малые градуировки}

Алгебры Ли $W_{1}$ и $L_{1}$ будем считать градуированными стандартным образом: $\operatorname{deg} e_{i}=i$. Мы рассматриваем градуированные модули над этими алгебрами и лишь те гомоморфизмы между такими модулями, которые являются однородными нулевой степени.

Рассмотрим естественное упорядочение на базисных элементах алгебры $W_{1}$ : $e_{i}>e_{j}$ при $i>j$. В модуле $S^{k} W_{1}$ рассмотрим стандартный мономиальный базис, образованный элементами $e_{i_{1}} e_{i_{2}} \cdots e_{i_{k}}\left(-1 \leqslant i_{1} \leqslant \cdots \leqslant i_{k}\right)$. Упорядочим этот базис следующим образом: отбросим все сомножители, равные $e_{-1}$, а на мономах $e_{i_{1}} e_{i_{2}} \cdots e_{i_{l}}$ с $l \leqslant k$ и $0 \leqslant i_{1} \leqslant \cdots \leqslant i_{l}$ введем лексикографическистепенной порядок: больше тот моном, у которого больше $l$, а при равных $l-$ бо́льший лексикографически; отметим, что здесь слово «степень» связано с «внешней» градуировкой (по числу образующих в мономе), в то время как в остальной части текста оно обычно отвечает «внутренней» градуировке (по сумме степеней образующих). В универсальной обертывающей алгебре $U=U\left(L_{1}\right)$ алгебры Ли $L_{1}$ можно по теореме Пуанкаре-Биркгофа-Витта (см. [3]) выбрать в качестве базиса совокупность элементов $E_{\alpha}:=e_{\alpha_{1}} e_{\alpha_{2}} \cdots e_{\alpha_{n}}$, занумерованных мультииндексами $\alpha=\left(\alpha_{1}, \ldots, \alpha_{n}\right)$, для которых $\left.0<\alpha_{1} \leqslant \alpha_{2} \leqslant \cdots \leqslant \alpha_{n}\right)$. На таких элементах также рассмотрим лексикографически-степенной порядок. Для элемента $f \in S^{k} W_{1}$ через $\widehat{f}$ мы обозначаем старший моном этого элемента относительно введенного на $S^{k} W_{1}$ порядка.

Лемма 1. Пусть $E_{\alpha}<E_{1^{k+1}}=e_{1}^{k+1}$. Тогда имеет место равенство

$$
\left.\widehat{E_{\alpha}\left(e_{-1}^{k}\right.}\right)=\frac{k !}{(k-n) !}(-1)^{n}\left(1+\alpha_{1}\right) \cdots\left(1+\alpha_{n}\right) e_{-1}^{k-n} e_{\alpha_{1}-1} \cdots e_{\alpha_{n}-1} \text {. }
$$

ДокАЗАТЕЛЬСтво состоит в прямом вычислении, основанном на том, что наибольшим из слагаемых будет слагаемое, содержащее наименьшее число вхождений $e_{-1}$.

СледСтвиЕ 1. Пусть $E_{\alpha}<E_{\beta}<e_{1}^{k+1}$. Тогда $\left.\widehat{E_{\alpha}\left(e_{-1}^{k}\right.}\right)<\widehat{E_{\beta}\left(e_{-1}^{k}\right)}$.

СлЕДСТвиЕ 2. Гомоморфизм $L_{1}$-модулей $\rho: U[-k] \rightarrow S^{k} W_{1}$, переводящий 1 в $e_{-1}^{k}$, сюоргективен. Он является изоморфизмом на однородных компоненmах степеней $-k, 1-k, \ldots, 0$ в $U[-k]$ и имеет одномерное ядро на однородной компоненте степени 1.

(Здесь и далее через $M[p]$ обозначается модуль, отличающийся от $M$ лишь сдвигом градуировки на $\left.p: M[p]_{i}:=M_{i-p}.\right)$

ДокАЗАТЕЛьСтво слЕДСтвия 2. Сюръективность можно доказать так: элементы $\rho\left(E_{\alpha}\right)=E_{\alpha}\left(e_{-1}^{k}\right)$, где $\alpha$ пробегает все мультииндексы $\alpha=\left(\alpha_{1}, \ldots, \alpha_{l}\right)$ длины $l \leqslant k$ (в этом случае $E_{\alpha}$ заведомо меньше $e_{1}^{k+1}$ и, значит, можно использовать следствие 1), имеют различные старшие мономы, причем среди этих 
мономов встречаются все базисные мономы симметрической степени $S^{k} W_{1}$ (согласно лемме 1, для элемента рассматриваемого вида старшим мономом будет, с точностью до константы, $e_{-1}^{k-l} e_{\alpha_{1}-1} \cdots e_{\alpha_{l}-1}$, и каждый базисный элемент симметрической степени имеет, очевидно, такой вид для подходящего выбора мультииндекса $\alpha$ ). Теперь очевидно, что каждый базисный моном принадлежит образу гомоморфизма $\rho$.

Утверждение об изоморфизме устанавливается аналогично: размерности однородных компонент степеней $-k, \ldots, 0$ в модуле $U[-k]$ такие же, как в симметрической степени $S^{k} W_{1}$ (поскольку для всех элементов базиса длина мультииндекса не превосходит $k$ ), а у компоненты степени 1 размерность больше на единицу (единственным элементом, для которого мультииндекс имеет бо́льшую длину, является $\left.e_{1}^{k+1}\right)$.

Для дальнейшего зафиксируем в $U[-k]$ однородный элемент $\phi$ степени 1 , для которого $\rho(\phi)=0$.

СледСТВиЕ 3. Существует свободная резолъвента $L_{1}$-модуля $S^{k} W_{1}$ вида

$$
0 \leftarrow S^{k} W_{1} \leftarrow U[-k] \leftarrow U[1] \oplus M^{1} \leftarrow M^{2} \leftarrow \cdots \leftarrow M^{i} \leftarrow \cdots .
$$

Здесъ все $M^{i}$ - свободные модули, причем у них отличны от нуля только однородные компоненты степени больше единищы.

ДокАзАтельство. Такая резольвента строится в соответствии с общей схемой построения минимальной резольвенты модуля. Гомоморфизм $\rho$ из $U[-k]$ в $S^{k} W_{1}$ определен выше. Он сюръективен, так что следующий шаг состоит в том, чтобы построить сюръекцию из свободного модуля на ядро этого гомоморфизма. Это ядро порождено элементом $\phi$ и какими-то элементами $\phi_{1}, \ldots, \phi_{s}, \ldots$ бо́льших степеней $n_{1}, \ldots, n_{s}, \ldots$ (конечно их количество или нет, неважно). Значит, на него отображается свободный модуль $U[1] \oplus \bigoplus_{s \geqslant 1} U\left[n_{s}\right]$ (гомоморфизм переводит единицу каждого из слагаемых в соответствующую образующую). Полагая $M^{1}:=\bigoplus_{s \geqslant 1} U\left[n_{s}\right]$, получаем первый модуль резольвенты. Дальнейшее построение проводится аналогично (надо все время иметь в виду, что ядра всех последующих гомоморфизмов содержат только элементы степени больше единицы, явный же вид этих гомоморфизмов для нас несуществен).

Как известно, гомологии алгебры Ли $\mathfrak{A}$ с коэффициентами в модуле $M$ можно вычислять двумя способами. Во-первых, эти гомологии совпадают с гомологиями стандартного комплекса $C_{*}(\mathfrak{A}, M)=\Lambda^{*}(\mathfrak{A}) \otimes M$. Во-вторых, в терминах универсальной обертывающей алгебры имеем $H_{*}(\mathfrak{A}, M)=\operatorname{Tor}_{*}^{U(\mathfrak{A})}(M, \mathbb{C})$. Во втором случае можно использовать полученные результаты о свободной резольвенте. Чтобы выписать результат, напомним, что гомологии градуированной алгебры Ли $\mathfrak{A}$ с коэффициентами в градуированном модуле $M$ наследуют градуировку; мы обозначаем через $H_{*}^{(p)}(\mathfrak{A}, M)$ соответствующую однородную компоненту.

ПРЕДЛОЖЕНИЕ 1. Имеют место равенства

$$
\begin{aligned}
H_{0}\left(L_{1}, S^{k} W_{1}\right) & =H_{0}^{(-k)}\left(L_{1}, S^{k} W_{1}\right)=\mathbb{C}, \\
H_{1}^{(\leqslant 1)}\left(L_{1}, S^{k} W_{1}\right) & =H_{1}^{(1)}\left(L_{1}, S^{k} W_{1}\right)=\mathbb{C}, \\
H_{>1}^{(\leqslant 1)}\left(L_{1}, S^{k} W_{1}\right) & =0 .
\end{aligned}
$$


Интересно получить сколько-нибудь явные формулы для циклов стандартного комплекса, представляющих найденные классы гомологий. Обозначим через $\psi_{k}$ единственный нетривиальный класс первых гомологий с коэффициентами в $S^{k} W_{1}$ степени 1 .

ПРЕДЛОЖЕНИЕ 2. В качестве иикла стандартного комплекса, представляющего $\psi_{k}$, можно выбрать элемент

$$
\sum_{i=0}^{k}(-1)^{i}\left(\begin{array}{c}
k+2 \\
i
\end{array}\right) e_{k+1-i} \otimes e_{-1}^{k-i} e_{0}^{i} .
$$

ДокАзАТЕльство. Тот факт, что дифференциал этого элемента равен нулю, проверяется непосредственно. Единственное, что представляет интерес, нетривиальность этого цикла. Проще всего использовать тот (очевидный) факт, что слагаемое $e_{1} \otimes e_{0}^{k}$ не может встретиться при вычислении дифференциала цепи стандартного гомологического комплекса.

Как известно, алгебра $L_{1}$ порождена элементами $e_{1}$ и $e_{2}$. Поэтому рассматриваемый цикл можно также выбрать в виде $e_{1} \otimes m_{1}+e_{2} \otimes m_{2}$. Приведем соответствующие формулы, поскольку они представляются достаточно любопытными. Основной ингредиент здесь - «оператор Казимира» $e_{-1} e_{1}-e_{0}^{2}$ для подалгебры $\mathfrak{s l}_{2} \subset W_{1}$, порожденной элементами $e_{-1}, e_{0}, e_{1}$.

ПРЕДЛОЖЕНИЕ 3. При $k=2 m$ можно в качестве представителя для $\psi_{k}$ взять

$$
e_{1} \otimes\left(e_{-1} e_{1}-e_{0}^{2}\right)^{m}
$$

при $k=2 m+1$ формула несколько более сложна:

$$
\begin{aligned}
e_{2} \otimes e_{-1}\left(e_{-1} e_{1}-e_{0}^{2}\right)^{m} & -(2 m+3) e_{1} \otimes e_{0}\left(e_{-1} e_{1}-e_{0}^{2}\right)^{m} \\
& +m e_{1} \otimes e_{-1}\left(e_{-1} e_{1}-e_{0}^{2}\right)^{m-1}\left(e_{-1} e_{2}-e_{0} e_{1}\right) .
\end{aligned}
$$

ДокАзАтЕльство. Доказательство нетривиальности этих циклов повторяет уже использованный выше аргумент: цикл, в который входит с ненулевым коэффициентом $e_{1} \otimes e_{0}^{k}$, не лежит в образе дифференциала.

\section{§3. Основная теорема}

Для вычисления гомологий алгебры Ли $W_{1}$ с коэффициентами в $S^{k} W_{1}$ мы используем спектральную последовательность Серра-Хохшильда. А именно, сначала вычислим гомологии относительно подалгебры $\mathfrak{g l}_{1}$, порожденной элементом $e_{0}$, с помощью относительной спектральной последовательности, а потом используем этот результат для доказательства основного результата работы.

Рассмотрим цепочку подалгебр $\mathfrak{g l}_{1} \subset L_{0} \subset W_{1}$. Применение общих формул для спектральной последовательности Серра-Хохшильда (см. [2]) в нашем случае дает спектральную последовательность с первым членом

$$
E_{p, q}^{1}=H_{q}\left(L_{0}, \mathfrak{g l}_{1}, \Lambda^{p}\left(W_{1} / L_{0}\right) \otimes S^{k} W_{1}\right),
$$

сходящуюся к $H_{*}\left(W_{1}, \mathfrak{g l}_{1}, S^{k} W_{1}\right)$. Вычислим пространство $E_{p, q}^{1}$ явно. Относительные гомологии вычисляются с помощью комплекса, аналогичного стандартному комплексу. В нашем случае при фиксированном $p$ это комплекс

$$
\left(\Lambda^{*}\left(L_{1}\right) \otimes \Lambda^{p}\left(W_{1} / L_{0}\right) \otimes S^{k} W_{1}\right)^{\mathfrak{g l}_{1}} .
$$


Легко видеть, что он отождествляется с подкомплексом стандартного комплекса, вычисляющего гомологии алгебры $L_{1}$ с коэффициентами в $\Lambda^{p}\left(W_{1} / L_{0}\right) \otimes$ $S^{k} W_{1}$, состоящим из элементов степени 0. С помощью результатов предыдущего параграфа мы можем получить полную информацию о нашей спектральной последовательности.

Лемма 2. Пространство $E_{1,1}^{1}$ совпадает с $\mathbb{C}$, все осталънъе пространства $E_{p, q}^{1}$ нулевъе.

ДоКАЗАТЕЛЬСТВо. Чтобы свести этот вопрос к вопросу, ответ на который известен, заметим, что имеет место изоморфизм $L_{1}$-модулей $W_{1} / L_{0} \simeq \mathbb{C}[-1]$ (тривиальный модуль с подправленной градуировкой). Поэтому $E_{1, q}^{1}=\left(E_{1, q}^{1}\right)^{(0)}=$ $H_{q}^{(1)}\left(L_{1}, S^{k} W_{1}\right)$. Кроме того, $E_{0, q}^{1}=\left(E_{0, q}^{1}\right)^{(0)}=H_{q}^{(0)}\left(L_{1}, S^{k} W_{1}\right)$. (Пространства $E_{p, q}^{1}$ при $p>1$ равны нулю по очевидным причинам.) Отсюда уже легко получить утверждение леммы.

СЛЕДСТВИЕ 4. $E_{p, q}^{n}=E_{p, q}^{1}$.

ДоКАЗАТЕЛьство. Это верно для любой спектральной последовательности, в которой ровно одно пространство $E_{p, q}^{1}$ ненулевое.

ДОКАЗАТЕЛЬСтво осНОВНОЙ тЕОРЕМЫ. Согласно следствию 4, пространство $E_{1,1}^{1}$ выживает в члене $E^{\infty}$ нашей спектральной последовательности. Поскольку спектральная последовательность сходится к интересующим нас гомологиям, первое утверждение теоремы доказано.

Второе утверждение доказывается с помощью рассмотрения спектральной последовательности Серра-Хохшильда относительно подалгебры $\mathfrak{g l}_{1}$. Первый член этой спектральной последовательности есть

$$
E_{p, q}^{1}=H_{q}\left(\mathfrak{g l}_{1}, \Lambda^{p}\left(W_{1} / \mathfrak{g l}_{1}\right) \otimes S^{k} W_{1}\right),
$$

и потому

$$
E_{p, 0}^{1}=E_{p, 1}^{1}=\left(\Lambda^{p}\left(W_{1} / \mathfrak{g l}_{1}\right) \otimes S^{k} W_{1}\right)^{\mathfrak{g l}_{1}}, \quad E_{p, k}^{1}=0 \quad \text { при } k>1 .
$$

Далее, гомологии дифференциала $d^{1}$ для каждого из значений $p=0,1$ совпадают, очевидно, с относительными гомологиями алгебры $W_{1}$ по модулю ее подалгебры $\mathfrak{g l}_{1}$. Значит, член $E^{2}$ этой спектральной последовательности отличен от нуля только в одном столбце. Поскольку дальнейшие дифференциалы $d^{k}$ отображают этот столбец в другие, все эти дифференциалы равны нулю. Поэтому $E^{2}=E^{\infty}$, и требуемое утверждение доказано.

\section{$\S 4$. Тензорные степени}

Естественным продолжением этого вычисления было бы вычисление гомологий с коэффициентами в тензорных степенях присоединенного представления. В полной общности эта задача весьма сложна. Здесь мы приводим результаты вычислений гомологий с коэффициентами в $W_{1}^{\otimes k}$ для $1 \leqslant k \leqslant 4$, некоторые гипотезы об общем случае, а также следствия из проделанных вычислений, касающиеся гомологий алгебры токов $W_{1} \otimes \mathbb{C}[t]$. Для того чтобы формулировки были проще, мы обсуждаем подробнее случай относительных гомологий по модулю подалгебры $\mathfrak{g l}_{1} \subset W_{1}$, порожденной элементом $e_{0}$.

Заметим, что имеется отображение гомологий

$$
H_{*}\left(\mathfrak{s l}_{2}, \mathfrak{g l}_{1}, \mathbb{C}\right) \otimes\left(\mathfrak{s l}_{2}^{\otimes n}\right)^{\mathfrak{s l}_{2}}=H_{*}\left(\mathfrak{s l}_{2}, \mathfrak{g l}_{1}, \mathfrak{s l}_{2}^{\otimes n}\right) \rightarrow H_{*}\left(W_{1}, \mathfrak{g l}_{1}, W_{1}^{\otimes n}\right),
$$


индуцированное вложением $\mathfrak{s l}_{2} \hookrightarrow W_{1}$. Гомологии алгебры $\mathfrak{s l}_{2}$ относительно $\mathfrak{g l}_{1}$, отличные от $H_{0}$ и $H_{2}$, равны нулю. Поэтому вопрос лишь в том, как устроено это отображение на нулевых и вторых гомологиях.

ПРЕДЛОЖЕНИЕ 4. На пространстве $H_{0}$ построенное отображсние равно нулю. На пространстве $\mathrm{H}_{2}$ это отображение является вложением.

Доказательство. Первое утверждение очевидно, ибо $H_{0}\left(W_{1}, \mathfrak{g l}_{1}, W_{1}^{\otimes n}\right)=0$. Рассмотрим пространство вторых гомологий. Пусть

$$
e_{-1} \wedge e_{1} \otimes \psi=d\left(\sum_{0<\alpha<\beta} e_{-1} \wedge e_{\alpha} \wedge e_{\beta} \otimes \psi_{\alpha \beta}+\sum_{0<\alpha<\beta<\gamma} e_{\alpha} \wedge e_{\beta} \wedge e_{\gamma} \otimes \psi_{\alpha \beta \gamma}\right) .
$$

Тогда $\psi=\sum_{1<\beta} e_{\beta}\left(\psi_{1 \beta}\right)$, что ведет к противоречию, ибо элемент $\psi$ является $\mathfrak{s l}_{2}$-инвариантом в $\mathfrak{s l}_{2}^{\otimes n}$, а $e_{\beta}\left(W_{1}^{\otimes n}\right) \cap\left(\mathfrak{s l}_{2}^{\otimes n}\right)^{\mathfrak{s l}_{2}}=0$ : при $\beta>2$ или $\beta=2$ и $n>2$ элементы из $e_{\beta}\left(W_{1}^{\otimes n}\right)$ не лежат даже в $\mathfrak{s l}_{2}^{\otimes n}$, при $\beta=n=2$ пересечение с тензорной степенью алгебры $\mathfrak{s l}_{2}$ одномерно и соответствующий элемент $e_{2}\left(e_{-1}^{2}\right)$ не инвариантен.

ЗАмЕчАНИЕ 1. Предположительно полученная нижняя оценка на размеры вторых гомологий почти точна. Разумеется, для $k=2 m+1$ основная теорема гарантирует, что $\operatorname{dim} H_{2}\left(W_{1}, \mathfrak{g l}_{1}, S^{k} W_{1}\right)=1$, в то время как $\operatorname{dim} H_{2}\left(\mathfrak{s l}_{2}, \mathfrak{g l}_{1}, S^{k} \mathfrak{s l}_{2}\right)$ $=0$. Заметим, однако, что все известные на данный момент циклы, не лежащие в образе построенного вложения, суть циклы $\psi_{2 k-1}(k=1,2, \ldots)$ для нечетных симметрических степеней.

Для $1 \leqslant k \leqslant 4$ явное вычисление показывает, что гомологии исчерпываются образом вложения из предложения 4 и гомологиями с коэффициентами в симметрических степенях. Результаты этого вычисления соединены в следующем предложении, доказательство которого является вычислительным и здесь не приводится.

ПРЕДЛОЖЕНИЕ 5. Пространства $H_{i}\left(W_{1}, \mathfrak{g l}_{1}, W_{1}^{\otimes k}\right)$ равнъь 0 при $i \neq 2,1 \leqslant$ $k \leqslant 4$. Кроме того,

$$
\begin{aligned}
H_{2}\left(W_{1}, \mathfrak{g l}_{1}, W_{1}\right) & =H_{2}\left(\mathfrak{s l}_{2}, \mathfrak{g l}_{1}, \mathfrak{s l}_{2}\right) \oplus H_{2}\left(W_{1}, \mathfrak{g l}_{1}, S^{1} W_{1}\right)=\mathbb{C}^{0} \oplus \mathbb{C}^{1}=\mathbb{C}, \\
H_{2}\left(W_{1}, \mathfrak{g l}_{1}, W_{1}^{\otimes 2}\right) & =H_{2}\left(\mathfrak{s l}_{2}, \mathfrak{g l}_{1}, \mathfrak{s l}_{2}^{\otimes 2}\right)=\mathbb{C}, \\
H_{2}\left(W_{1}, \mathfrak{g l}_{1}, W_{1}^{\otimes 3}\right) & =H_{2}\left(\mathfrak{s l}_{2}, \mathfrak{g l}_{1}, \mathfrak{s l}_{2}^{\otimes 3}\right) \oplus H_{2}\left(W_{1}, \mathfrak{g l}_{1}, S^{3} W_{1}\right)=\mathbb{C}^{1} \oplus \mathbb{C}^{1}=\mathbb{C}^{2}, \\
H_{2}\left(W_{1}, \mathfrak{g l}_{1}, W_{1}^{\otimes 4}\right) & =H_{2}\left(\mathfrak{s l}_{2}, \mathfrak{g l}_{1}, \mathfrak{s l}_{2}^{\otimes 4}\right)=\mathbb{C}^{3} .
\end{aligned}
$$

СЛЕДСТВИЕ 5. Пространства $H_{i}\left(W_{1}, W_{1}^{\otimes k}\right)$ равны 0 nрu $i \neq 2,3,1 \leqslant k \leqslant 4$. Кроме того,

$$
\begin{array}{cl}
H_{2}\left(W_{1}, W_{1}\right)=H_{3}\left(W_{1}, W_{1}\right)=\mathbb{C}, & H_{2}\left(W_{1}, W_{1}^{\otimes 2}\right)=H_{3}\left(W_{1}, W_{1}^{\otimes 2}\right)=\mathbb{C}, \\
H_{2}\left(W_{1}, W_{1}^{\otimes 3}\right)=H_{3}\left(W_{1}, W_{1}^{\otimes 3}\right)=\mathbb{C}^{2}, & H_{2}\left(W_{1}, W_{1}^{\otimes 4}\right)=H_{3}\left(W_{1}, W_{1}^{\otimes 4}\right)=\mathbb{C}^{3} .
\end{array}
$$

ГиПОТЕЗА. При $i>2$ и любом $k$ пространства $H_{i}\left(W_{1}, \mathfrak{g l}_{1}, W_{1}^{\otimes k}\right)$ равнъц 0 ; при $i>3$ и любом $k$ пространства $H_{i}\left(W_{1}, W_{1}^{\otimes k}\right)$ равнъь 0 .

У тверждения предложения 5 и следствия из него позволяют предположить, что можно заменить в приведенной гипотезе условие $i>2$ (соответственно $i>3$ ) на $i \neq 2$ (соответственно $i \neq 2,3)$. Оказывается, что этого сделать 
нельзя: для $W_{1}^{\otimes 5}$ первые гомологии отличны от нуля. В этом можно убедиться следующим образом. Рассмотрим действие симметрической группы $S_{5}$ на тензорной степени $W_{1}^{\otimes 5}$ перестановками сомножителей. Разложение тензорной степени на изотипные компоненты известно - это представление симметрической группы, очевидно, изоморфно представлению в кольце многочленов от пяти переменных (и градуировки на соответствующих векторных пространствах отличаются только сдвигом), а результаты о разложении этого представления в сумму неприводимых являются стандартными. Для представления группы $S_{5}$, отвечающего разбиению $5=2+1+1+1$, минимальная степень однородных многочленов от пяти переменных, в которых реализуется такое представление, равна 6 (и в многочленах степени 6 такое представление встречается с кратностью один). Для $W_{1}^{\otimes 5}$ после переградуировки получаем, что такое представление встречается первый раз в степени 1, причем с кратностью один. Обозначим это вхождение через $V$. Тогда любой элемент пространства $e_{-1} \otimes V$ будет, как легко видеть, циклом стандартного комплекса (и относительного, и обычного), причем нетривиальным (раз в меньших степенях такое представление не встречается).

Информация о гомологиях с коэффициентами в тензорных степенях полезна тем, что ее можно переработать в информацию о гомологиях алгебры (полиномиальных) токов на прямой со значениями в алгебре $W_{1}$. Напомним, что алгеброй полиномиальных токов на прямой со значениями в алгебре Ли $\mathfrak{A}$ называется пространство $\mathfrak{A} \otimes \mathbb{C}[t]$ со скобкой $[a \otimes f, b \otimes g]=[a, b] \otimes(f g)$. Вычисление проводится с помощью спектральной последовательности Серра-Хохшильда для подалгебры $W_{1} \otimes 1$. Мы приводим здесь без доказательства результаты вычисления гомологий относительно подалгебры $\mathfrak{g l}_{1}:=\mathbb{C} e_{0} \otimes 1 \subset W_{1} \otimes 1 \subset W_{1} \otimes \mathbb{C}[t]$.

Полученные результаты удобно формулировать так. Алгебра Ли векторных полей на прямой с координатой $t$ действует на алгебре токов по очевидному правилу $\xi(a \otimes f)=a \otimes \xi(f)$. Это действие обычным образом переносится на любую тензорную конструкцию, например на внешнюю степень, и потому на весь стандартный комплекс. Очевидно, что это действие перестановочно с дифференциалом стандартного комплекса и потому алгебра векторных полей на прямой действует на гомологиях.

ПРЕДЛОЖЕНИЕ 6. Имеют место следующие равенства:

$$
\begin{array}{ll}
H_{0}\left(W_{1} \otimes \mathbb{C}[t], \mathfrak{g l}_{1}, \mathbb{C}\right)=\mathbb{C}, & H_{1}\left(W_{1} \otimes \mathbb{C}[t], \mathfrak{g l}_{1}, \mathbb{C}\right)=0, \\
H_{2}\left(W_{1} \otimes \mathbb{C}[t], \mathfrak{g l}_{1}, \mathbb{C}\right)=\mathbb{C}, & H_{3}\left(W_{1} \otimes \mathbb{C}[t], \mathfrak{g l}_{1}, \mathbb{C}\right)=\mathbb{C}[t] d t, \\
H_{4}\left(W_{1} \otimes \mathbb{C}[t], \mathfrak{g l}_{1}, \mathbb{C}\right)=0, & H_{5}\left(W_{1} \otimes \mathbb{C}[t], \mathfrak{g l}_{1}, \mathbb{C}\right)=S^{3}(\mathbb{C}[t] d t) .
\end{array}
$$

Указанные равенства нужно понимать как изоморфизмы модулей над алгеброй Ли векторных полей на прямой.

\section{ЛитерАтУрА}

1. Feigin B. L., Tsygan B. L. Riemann-Roch theorem and Lie algebra homology. In: Proc. of the Winter School on Geometry and Physics (Srni, 9-16 January 1988), Rend. Circ. Mat. Palermo (2) Suppl., No. 21, 15-52 (1989).

2. Фукс Д. Б. Когомологии бесконечномерных алгебр Ли. Наука, М., 1984.

3. Картан А., Эйленберг С. Гомологическая алгебра. ИЛ, М., 1960. 